\title{
Composição e percepção corporal de adolescentes de escolas públicas
}

\author{
Body composition and perception of teenagers from public schools
}

\author{
A.A. Ferreira, J.D. Nogueira, I. Wiggers, K.E. Fontana
}

ARTIGO ORIGINAL | ORIGINAL ARTICLE

\begin{abstract}
A adolescência é acompanhada de mudanças cognitivas, emocionais, sociais e biológicas que ampliam as situações de risco ao desenvolvimento de disfunções psicossomáticas. Este estudo aferiu e classificou a composição corporal e a comparou à autopercepção corporal em adolescentes. Alunos do sétimo ao nono ano do ensino fundamental público no Distrito Federal, Brasil, responderam a questionários sóciodemográfico e de percepção corporal. Massa e estatura foram medidas e o Índice de Massa Corporal (IMC) foi calculado para classificação da composição corporal. Dos 977 adolescentes, $79.1 \%$ apresentaram IMC eutrófico. Dos 473 meninos, $11.4 \%$ apresentaram excesso de peso e $4.7 \%$ baixo peso; $23.8 \%$ perceberam o corpo como menor do que realmente é e $25.5 \%$ tentaram ganhar massa corporal. Das 504 meninas, $11.9 \%$ apresentaram excesso de peso e $13.4 \%$ baixo peso; $24.1 \%$ perceberam o corpo como maior do que realmente é e $32.5 \%$ tentaram perder massa corporal. A composição corporal inadequada, prevalente em $20.9 \%$ dos adolescentes, pode trazer prejuízos ao crescimento, desenvolvimento e saúde. Estes problemas podem ser agravados pela elevada prevalência de autopercepção corporal distorcida e de atitudes para obter mudanças corporais. Recomenda-se a realização de intervenções educativas sobre composição, percepção e cultura corporal e saúde de forma integrada, com abordagens diferenciadas por sexo.

Palavras-chave: imc, imagem corporal, sobrepeso, baixo peso, saúde
\end{abstract}

ABSTRACT

Adolescence is accompanied by cognitive, emotional, social and biological changes; situations that increase the risk for development of psychosomatic disorders. This study measured and classified body composition and compared it to body self-perception in adolescents. Students from the seventh to ninth grade in public primary education in Distrito Federal, Brazil, answered socio-demographic and body self-perception questionnaires. Weight and height were measured and body mass index (BMI) was calculated for body composition classification. From the 977 adolescents, $79.1 \%$ presented eutrophic BMI. Of the 473 boys, $11.4 \%$ were overweight and $4.7 \%$ underweight, $23.8 \%$ perceived the body as smaller than it really is and $25.5 \%$ tried to gain body mass. Of the 504 girls, $11.9 \%$ were overweight and $13.4 \%$ underweight, $24.1 \%$ perceived the body as larger than it really is and $32.5 \%$ tried to lose body mass. Inadequate body composition, prevalent in $20.9 \%$ of adolescents, may harm growth, development and health. These problems may be aggravated by the high prevalence of distorted body self-perception and attitudes for bodily changes. It is recommended the implementation of educational interventions on body composition, perception and culture and health, with different approaches by gender.

Keywords: bmi, body image, overweight, underweight, health

Submetido: 20.03.2012 | Aceite: 26.12.2012

Ana Paula Araújo Ferreira, Júlia Aparecida Devidé Nogueira, Ingrid Wiggers, Keila Elizabeth Fontana. Faculdade de Educação Física, Universidade de Brasília, Brasil.

Endereço para correspondência: Júlia Aparecida Devidé Nogueira, Faculdade de Educação Física,

Campus Universitário Darcy Ribeiro, Brasília-DF, CEP: 70.000-000, Brasil.

E-mail: julianogueira@unb.br 
Nas últimas décadas, a prevalência de sobrepeso e obesidade tem aumentado de forma preocupante em todo o mundo (Wang, Monteiro, \& Popkin, 2002). A Organização Mundial da Saúde (OMS) define sobrepeso como excesso de massa corporal em comparação com tabelas ou padrões de normalidade e obesidade como doença decorrente do excesso de gordura corporal que acarreta prejuízos à saúde, qualidade e quantidade de vida (OMS, 2000).

O Ministério da Saúde (MS) aponta que, desde 1960, o Brasil enfrenta mudanças no padrão alimentar da população que, juntamente com o aumento do sedentarismo, comportamento típico da vida moderna, resulta no aumento do sobrepeso e da obesidade (MS, 2006). De 2006 a 2009, a proporção de brasileiros com excesso de peso subiu de $42.7 \%$ para $46.6 \%$ e a de obesos cresceu de $11.4 \%$ para $13.9 \%$ (MS, 2009).

Países em desenvolvimento enfrentam, além das doenças causadas pelo excesso de peso, uma dupla carga de doenças pela existência concomitante da desnutrição, decorrente da ingestão energética e de nutrientes em quantidades inferiores às necessidades do organismo. No Brasil, entre 1970 e 1989, a prevalência da desnutrição sofreu redução de $19.8 \%$ para $7.6 \%$ (Mondini \& Monteiro, 1999), mas ainda não foi erradicada.

O desenvolvimento de estados nutricionais inadequados também tem afetado crianças e adolescentes. Atualmente, cerca de um quarto das crianças americanas e brasileiras apresentam sobrepeso ou obesidade (Ogden, Carroll, Curtin, McDowell, Tabak, \& Flegal, 2006; Wang et al., 2002). Esta situação preocupante é agravada pelo fato de que doenças crônicas não transmissíveis (DCNT), como hipertensão, diabetes tipo 2 e hipercolesterolemia, que em geral afetam adultos e idosos, têm atingido cada vez mais a população jovem (Weiss \& Kaufman, 2008).

A adolescência é um período no qual o indi- víduo em formação experimenta importantes mudanças cognitivas, emocionais, sociais e biológicas, fundamentais para a determinação de sua saúde (Palacios, 1995). Nessa etapa também ocorre grande parte do desenvolvimento físico, quando o adolescente adquire em torno de $25 \%$ da estatura e $50 \%$ da massa corporal final, ficando exposto a mudanças indesejadas na composição corporal decorrentes da alteração na proporção de água, minerais e proteínas (Roemmich, Clark, Weltman, \& Rogol, 1997). Estudos reportam ainda que os estilos de vida adotados por adolescentes não são saudáveis, havendo elevada prevalência de inatividade física e consumo insuficiente de frutas e verduras, o que dificulta a manutenção da composição corporal adequada (Dowdell \& Santucci, 2004; Kann, Kinchen, Williams, Ross, Lowry, Grunbaum, \& Kolbe, 2000).

Atenta a esse contexto, a OMS (2000) preconiza que pesquisas com crianças e adolescentes utilizem medidas antropométricas para avaliar o estado nutricional, possibilitando a detecção precoce de disfunções orgânicas, estados de subnutrição ou de excesso de peso e fatores de risco para desenvolvimento de DCNT. O estado nutricional pode ser classificado com base na composição corporal através do Índice de Massa Corporal (IMC). O IMC é um método bastante aceito cientificamente devido à facilidade de sua aplicação e por apresentar relação com a gordura corporal total, mesmo em populações jovens. Os pontos de corte para classificação do baixo peso, sobrepeso e obesidade devem ser estabelecidos através de amostras representativas do país, adequadas à faixa etária e sexo (Cole, Bellizzi, Flegal, \& Dietz, 2000; Cole, Flegal, Nicholls, \& Jackson, 2007).

Entretanto, cabe ressaltar que, além da composição corporal em si, é importante considerar a autopercepção ou imagem corporal desenvolvida pelo indivíduo. A percepção corporal é uma manifestação humana multidimensional e complexa, que envolve aspectos cognitivos, afetivos, sociais, culturais e 
motores, incluindo a competência esportiva, condição física, aparência e força física (Silva \& Palmeira, 2010). Essa percepção é formada por intermédio das relações mútuas entre o organismo e o meio que o cerca, e pode representar o núcleo central da estruturação da personalidade (Hagger, Biddle, \& Wang, 2005).

A relação entre composição corporal e percepção mental do próprio corpo se justifica porque esta última ultrapassa a realidade neuropsicológica, oferecendo um conceito mais complexo para a abordagem de estudos sobre o corpo, englobando, além do aspecto mental, a dimensão social (Silva \& Palmeira, 2010). A busca de uma imagem corporal baseada em modelos estereotipados de corpo é um fenômeno bastante presente na sociedade brasileira atual, podendo apresentar reflexos na composição corporal e saúde dos indivíduos (Adami, Fernandes, Frainer, \& Oliveira, 2005).

Este estudo classificou a composição corporal por meio do IMC e comparou os valores aferidos à autopercepção corporal de adolescentes de escolas públicas do Distrito Federal (DF), Brasil.

\section{MÉTODO}

\section{Amostra}

Todos os alunos do sétimo ao nono ano do ensino fundamental de três Centros de Ensino Fundamental (CEF) do Distrito Federal, Região Centro-Oeste do Brasil foram convidados a participar da pesquisa, sendo incluídos aqueles com idade entre dez e dezoito anos que assinaram o termo de consentimento livre e esclarecido. Participaram do estudo 977 adolescentes, $51.6 \%$ do sexo feminino e $48.4 \%$ do sexo masculino, que tinham entre dez e dezoito anos (14.07 \pm 1.45 anos).

\section{Instrumentos e Procedimentos}

Os dados foram coletados nas escolas durante o horário letivo regular, permitindo elevada participação dos estudantes. Inicialmente, os adolescentes responderam a um questionário com questões sobre idade, sexo, maturação sexual (presença de pelos axilares para meninos e menarca para meninas), autopercepção corporal atual ("muito acima do peso", "acima do peso", "peso normal”, "abaixo do peso" ou "muito abaixo do peso"), e o que fazem em relação a seu corpo ("nada, tenta manter o peso", "tenta emagrecer" ou "tenta ganhar peso").

A seguir, os adolescentes tiveram sua massa e estatura corporais aferidas conforme o manual de referência para medidas antropométricas (Lohman, Roche, \& Martorell, 1988). Foi utilizado balança digital marca "Plenna" com capacidade de $150 \mathrm{~kg}$ e precisão de 0.1 $\mathrm{kg}$ e estadiômetro marca "WCS" com $212 \mathrm{~cm}$ e precisão de $0.5 \mathrm{~cm}$. Os valores de massa e estatura corporal foram usados para cálculo do IMC $\left(\mathrm{kg} / \mathrm{m}^{2}\right)$. O IMC foi comparado a pontos de corte de acordo com o sexo e a idade (Cole et al., 2000; Cole et al., 2007) para classificar a composição corporal.

\section{Análise Estatística}

Os resultados são apresentados como média e desvio-padrão ( $\mathrm{M} \pm \mathrm{DP})$ e prevalência (\%) de ocorrências. Diferenças estatísticas nas variáveis por sexo foram averiguadas por intermédio do Teste $t$ de student não pareado e, nas prevalências, por meio do Teste Qui-quadrado, utilizando o programa SPSS versão 7.0 (SPSS Inc., Chicago, EUA). O valor de significância considerado foi $p<.05$.

\section{RESULTADOS}

Dos 977 adolescentes estudados, 69\% afirmou ter os sinais de maturação e $74.2 \%$ indicou que seus pais cursaram no máximo o ensino médio, sem diferença estatisticamente significativa entre os sexos.

Ao avaliar a composição corporal por meio do IMC, a maior parte dos adolescentes (79.1\%) foram considerados eutróficos; $11.7 \%$ foram considerados com excesso de peso e $9.2 \%$ com baixo peso. A classificação da composição 
corporal dos adolescentes pelos pontos de corte do IMC, separados por sexo, é apresentada na tabela 1 .

Ao comparar a classificação da composição corporal obtida pelo IMC com a percepção corporal que os adolescentes têm de si mesmos, verificou-se que $64.1 \%$ deles percebem corretamente sua composição corporal, $18.9 \%$ percebem-se menores e $17.0 \%$ percebem-se maiores do que realmente são. A comparação da autopercepção corporal com o IMC medido, separada por sexo, está na tabela 2.

Ao investigar o comportamento dos adolescentes quanto a atitudes que estariam adotando em relação a seu peso, $52.9 \%$ relataram tentar manter ou não fazer nada, $21.5 \%$ relataram tentar emagrecer, e $25.5 \%$ relataram tentar ganhar peso. Estes comportamentos relatados pelos adolescentes, separados por sexo, são apresentados na tabela 3 .

Complementarmente, uma revisão bibliográfica sobre a prevalência do estado nutricional de adolescentes brasileiros foi realizada para subsidiar a comparação e discussão dos resultados encontrados no presente estudo. As bases de dados PubMed, Lilacs, Cochrane, Scielo e BVS foram pesquisadas com diferentes combinações das palavras chaves (em português e inglês): IMC, estado nutricional, composição corporal, escolares, adolescentes e Brasil. Dados referentes aos estudos publicados entre os anos 2000 a 2010 compuseram o Quadro 1. Para os meninos, a prevalência de excesso de peso foi maior nas populações de maior renda e/ou nas regiões Sul e Sudeste do Brasil, que possuem maior Índice de Desenvolvimento Humano (IDH). Em contraste, para as meninas, a prevalência de excesso de peso foi maior nas regiões brasileiras com menor IDH.

\section{DISCUSSÃO}

O presente estudo estimou a prevalência das classificações da composição corporal baseadas no IMC e as comparou com aquelas obtidas por meio da autopercepção corporal, em adolescentes de escolas públicas de Santa Maria,

\section{Tabela 1}

Prevalência das classificações da composição corporal dos adolescentes pelos pontos de corte do Índice de Massa Corporal (IMC), separados por sexo

\begin{tabular}{cccc}
\hline Sexo & IMC eutrófico (\%) & IMC baixo peso (\%) & IMC excesso de peso (\%) \\
\hline Feminino $(n=504)$ & 74.8 & 13.4 & 11.9 \\
Masculino $(n=473)$ & $83.9^{*}$ & $4.7^{*}$ & 11.4 \\
\hline
\end{tabular}

${ }^{*} p<.05$, significativamente diferente entre os sexos (Teste Qui-quadrado)

Tabela 2

Prevalência da percepção da composição corporal comparada ao IMC medido em adolescentes, separados por sexo

\begin{tabular}{cccc}
\hline \multirow{2}{*}{ Sexo } & $\begin{array}{c}\text { Percebe composição } \\
\text { corporal de maneira } \\
\text { similar à medida no IMC } \\
(\%)\end{array}$ & $\begin{array}{c}\text { Percebe composição } \\
\text { corporal como menor do } \\
\text { que foi medido no IMC }\end{array}$ & $\begin{array}{c}\text { Percebe composição } \\
\text { corporal como maior do } \\
\text { que foi medido no IMC } \\
(\%)\end{array}$ \\
\hline Feminino $(n=504)$ & 61.6 & $1 \%)$ & 24.1 \\
Masculino $(n=473)$ & $66.8^{*}$ & $23.8^{*}$ & $9.3^{*}$ \\
\hline
\end{tabular}

${ }^{*} p<.05$, significativamente diferente entre os sexos (Teste Qui-quadrado)

Tabela 3

Prevalência do comportamento dos adolescentes quanto à manutenção, ganho ou perda de massa corporal, separados por sexo

\begin{tabular}{cccc}
\hline Sexo & $\begin{array}{c}\text { Tenta manter a massa } \\
\text { corporal, não faz nada }(\%)\end{array}$ & $\begin{array}{c}\text { Tenta ganhar massa } \\
\text { corporal }(\%)\end{array}$ & $\begin{array}{c}\text { Tenta perder massa } \\
\text { corporal }(\%)\end{array}$ \\
\hline Feminino $(n=504)$ & 49.6 & 17.9 & 32.5 \\
Masculino $(n=473)$ & $56.5^{*}$ & $25.5^{*}$ & $18.0^{*}$ \\
\hline
\end{tabular}

${ }^{*} p<.05$, significativamente diferente entre os sexos (Teste Qui-quadrado) 


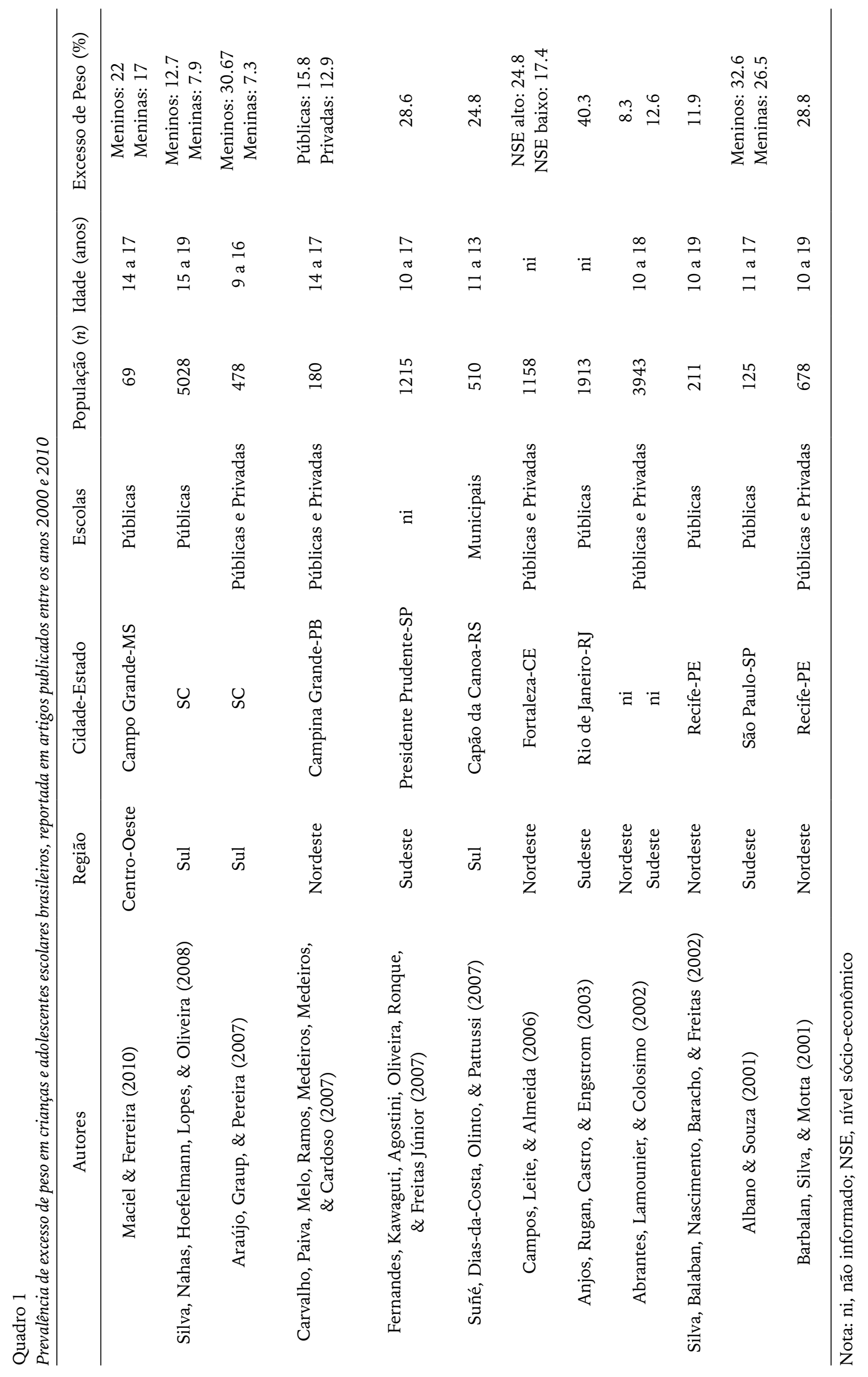


DF, Brasil. Segundo o Governo do Distrito Federal (GDF), a região localizada na periferia de Brasília abriga atualmente cerca de $130 \mathrm{mil}$ habitantes, sendo que $60 \%$ das famílias declararam ter renda mensal entre um a cinco salários mínimos (GDF, 2009).

A maioria dos escolares estudados foi classificada pelo IMC como eutrófica. Entretanto, $20.9 \%$ deles não apresentam proporções saudáveis de massa em relação à estatura, adequadas à sua faixa etária e sexo. Outros trabalhos listados no quadro 1 também indicam significativa prevalência de excesso de peso em adolescentes brasileiros. Tanto o baixo peso quanto o excesso de peso podem prejudicar o crescimento, desenvolvimento e a saúde destes adolescentes (Oliveira \& Fisberg, 2003; Weiss \& Kaufman, 2008).

O excesso de peso na infância está associado a prejuízos futuros à saúde. Cerca de $50 \%$ das crianças obesas aos sete anos de idade serão adultos obesos e cerca de $80 \%$ dos adolescentes obesos se tornarão adultos obesos (Billington \& Levine, 2000). No âmbito biológico, o excesso de peso aumenta o risco para desenvolver DCNT (OMS, 2000; Weiss \& Kaufman, 2008), resultando na diminuição da qualidade e expectativa de vida dos indivíduos que convivem com a obesidade por períodos prolongados (Ogden et al., 2006; Wieting, 2008). Com relação aos aspectos afetivos, psicológicos e comportamentais, as consequiências do excesso de peso podem ser baixa autoestima, isolamento, dificuldade de aprendizagem, agressividade, depressão e até o suicídio (Murphy, Horton, Burke, Monson, Laird, Lesage, \& Sobol, 2009; Sisto, 2005).

Concomitantemente, a desnutrição infantil ainda é um problema de saúde pública em algumas regiões do Brasil. Entretanto, a maioria dos estudos não reporta mais sua prevalência, provavelmente devido aos declínios substanciais em seus índices a partir de 1950 (Mondini \& Monteiro, 1998). Adolescentes participantes da presente pesquisa, principalmente as meninas, apresentaram prevalência significativa de baixo peso, o que pode resultar em crescimento e desenvolvimento deficientes, maior vulnerabilidade a doenças infecciosas, comprometimento de funções reprodutivas e redução da capacidade de trabalho na vida adulta (OMS, 2000; Wang et al., 2002).

Cabe ressaltar que a vulnerabilidade a distúrbios no estado nutricional é marcadamente determinada pela classe social, pelo sexo e pela faixa etária (Mondini \& Monteiro, 1998). Dados do presente estudo e os compilados no quadro 1 também apontam relação de aspectos socioeconômicos e sexo com a composição corporal.

Em adição, os dados desta pesquisa evidenciaram que a proporção de adolescentes que não possuem uma correta autopercepção corporal (35.9\%) foi ainda maior que a prevalência de adolescentes que efetivamente apresentaram composição corporal inadequada (20.9\%).

Algumas teorias ajudam a explicar as razões da discrepância entre a composição corporal real e a percebida. A autopercepção corporal é uma espécie de aprendizagem que pode ser compreendida como a imagem do corpo interpretada pela mente, ou seja, o modo como o corpo se apresenta para o indivíduo, mediado por sensações e experiências (Hagger et al., 2005). O indivíduo pode formar dois tipos de imagem corporal: as reprodutivas, que são reflexo da realidade objetiva e contribuem para a estruturação do real; e as antecipadoras ou alucinatórias, geradas pela ação da afetividade e do caráter subjetivo do indivíduo e tendem a afastar a pessoa da realidade (Bachelard, 1996). Além disso, o conhecimento do corpo é aprendido a cada dia ao longo da vida, ou seja, à medida que a criança cresce e se desenvolve, modifica sua percepção corporal (Palacios, 1995).

Os resultados obtidos neste trabalho mostram que os meninos se sentem menores do que realmente são e buscam um corpo mais forte, e as meninas se sentem maiores do que 
são e almejam um corpo magro. Sabe-se que os indivíduos fazem o julgamento do próprio corpo com base em influências socioculturais (Adami et al., 2005; Silva \& Palmeira, 2010).

Os padrões de corpo masculino e feminino difundidos atualmente como modelos ideais de aceitação e de êxito social se consolidaram a partir de 1980, por meio da generalização das práticas de body-building nos Estados Unidos, uma das manifestações contemporâneas mais relevantes da cultura do corpo (Courtine, 1995). A partir deste período, diversas esferas sociais, destacadamente a indústria, o mercado e, sobretudo um conjunto de práticas sociais de massa vêm aperfeiçoando as formas de divulgação desses ideais de corpo. Hoje, a imagem do corpo ideal é protagonizada por celebridades e divulgada pela publicidade, espetáculos esportivos na mídia, brinquedos, revistas, programas de televisão, obras literárias, entre outros (Courtine, 1995), mesmo para crianças, como é o caso das "bonecas-manequim" estilo Barbie (Brougère, 2000). Sob influência desse conceito, crianças aprendem desde cedo a valorizar a aparência física (Pinheiro \& Giugliani, 2006). Na adolescência, essa preocupação tende a aumentar devido aos efeitos emocionais e físicos da maturação sexual.

A ampla divulgação dos estereótipos de corpo ideal faz com que a insatisfação com a autopercepção corporal não fique restrita aos indivíduos que apresentam alterações clínicas na composição corporal, como foi o caso dos adolescentes do DF. Outro estudo, com crianças entre dez e doze anos, 25 obesas e 27 não obesas, observou que ambos os grupos de crianças manifestaram não estar satisfeitas com seu corpo, embora as crianças não obesas tenham expressado menor preocupação (Carvalho, Cataneo, Galindo, \& Malfará, 2005).

Esses resultados ressaltam a importância do aporte psicológico e de informação que cada criança possui para a formação da sua imagem corporal. Ao construir uma idéia ou imaginar um objeto, a pessoa não atua puramente com um dispositivo de percepção; há sempre uma personalidade que experimenta essa percepção (Schilder, 1999). Assim, especialmente na infância e na adolescência, é importante que educadores compreendam este fenômeno e atuem no sentido de educar para que cada aluno desenvolva uma percepção adequada de seu corpo.

Ao assumir e se identificar com um corpo em constante mudança, esteja ou não associada à obesidade ou sobrepeso, o adolescente pode tentar provocar alterações em sua composição corporal. O estudo em tela indica elevada prevalência $(47.1 \%)$ de adolescentes que relataram realizar atitudes para modificar a composição corporal, mesmo entre aqueles que foram classificados na faixa adequada à idade e ao sexo.

Essas tentativas se dão, muitas vezes, por meio de modificações no comportamento alimentar (Braggion, Matsudo, \& Matsudo, 2000; Nunes, Olinto, Barros, \& Camey, 2001), o que amplia as chances do desenvolvimento de transtornos alimentares (Bosi \& Andrade, 2004) e conseqüentes prejuízos à saúde (OMS, 2000). Atualmente, o transtorno alimentar com maior risco de morte entre adolescentes é a anorexia, caracterizada pela acentuada perda de peso auto-induzida, devido ao receio de engordar e a distorção da auto-imagem corporal (Bosi \& Andrade, 2004).

Embora não se possa afirmar que a distorção da autopercepção corporal e a insatisfação com a composição corporal evidenciadas nos adolescentes estudados estejam produzindo comportamentos insalubres, é provável que parte destes adolescentes esteja realizando dietas sem uma adequada orientação para modificar sua composição corporal. Esta hipótese é reforçada pela indicação de que adolescentes tendem a diminuir seu nível de atividade física (Braggion et al., 2000; Hills, King, \& Armstrong, 2007), e que a maioria da população jovem não realiza o mínimo de atividade física recomendada para manter uma composição corporal saudável (Sallis, Prochaska, \& Taylor, 2000). 
Em contrapartida, outras pesquisas com adolescentes mostram que a prática de atividade física está diretamente associada a índices mais elevados de autoconceito corporal (Adami et al., 2005; Moreno \& Cervelló, 2005) e motivação (Silva \& Palmeira, 2010). Sobretudo entre os jovens, a prática de atividade física pode promover o desenvolvimento integral, produzindo benefícios psicossociais e fisiológicos (Silva, Nahas, Hoefelmann, Lopes, \& Oliveira, 2008; Silva \& Palmeira, 2010). Tais constatações ganham relevância porque a prática da atividade física iniciada na infância ou na adolescência tende a se manter na vida adulta (Tassitano, Bezerra, Tenório, Colares, Barros, \& Hallal, 2007).

Com base nesta discussão, o presente estudo sugere análises mais abrangentes do que aquelas sobre alterações na composição corporal centradas apenas na obesidade e suas conseqüências físicas ao crescimento, desenvolvimento e saúde de adolescentes. Considerando o entendimento de que a percepção corporal distorcida pode agravar os prejuízos físicos, gerar danos psicológicos e promover a adoção de comportamentos insalubres, recomenda-se a realização de intervenções educativas sobre composição, percepção e cultura corporal, e saúde de forma integrada, com abordagens diferenciadas por sexo.

Como subsídio para futuras pesquisas, cabe destacar o papel da escola neste processo. Por exemplo, aulas de educação física podem ter seu escopo ampliado, pois ao mesmo tempo em que provocam efeitos na composição corporal podem oferecer instrumentos para a formação saudável da percepção corporal, pois a vivência de jogos, ginástica, esportes, lutas, brincadeiras e outras atividades são uma oportunidade de mediação e experiência corporal significativa. Além disso, podem contribuir com a leitura crítica do contexto social, sobretudo de mensagens que enfatizam estereótipos de corpo. Diante do quadro de pressões socioculturais relacionadas ao corpo e, paradoxalmente, de inatividade física e má alimentação por parte de adolescentes, a escola pode representar a principal rota para a comunicação em grande escala com esse grupo social.

\section{CONCLUSÕES}

Em suma, o presente estudo ressaltou que não apenas a obesidade, mas também o baixo peso e a insatisfação corporal são problemas que merecem atenção na adolescência, evidenciando interfaces entre o corpo físico e o corpo percebido. Nesse sentido, a prática educativa deve se orientar por objetivos relacionados ao controle da composição corporal, mas que também considerem outras dimensões correlatas e não menos importantes, como a nutrição, a atividade física, a saúde, a percepção e a cultura corporal, com abordagens diferenciadas por sexo.

Agradecimentos:

Nada declarado.

\section{Conflito de Interesses:}

Nada declarado.

\section{Financiamento:}

Apoio financeiro do CNPq - Conselho Nacional de Desenvolvimento Científico e Tecnológico Edital MCT-CNPq 51/2005.

\section{REFERÊNCIAS}

Abrantes, M. M., Lamounier, J. A., \& Colosimo, E. A. (2002). Prevalência de sobrepeso e obesidade em crianças e adolescentes das regiões Sudeste e Nordeste. Jornal de Pediatria, 78(4), 335-340. doi: 10.2223/JPED.869

Adami, F., Fernandes, T. C., Frainer, D. E. S., \& Oliveira, F. R. (2005). Aspectos da construção e desenvolvimento da imagem corporal e implicações na Educação Física. Revista Digital de Buenos Aires, 83. Consultado em 10 de outubro de 2009, a partir de http://www.efdeportes.com/ efd83/imagem.htm.

Albano, R. D., \& Souza, S. B. (2001). Estado 
nutricional de adolescentes: "risco de sobrepeso" e "sobrepeso" em uma escola pública do município de São Paulo. Caderno de Saúde Pública, 17(4), 941-947. doi: 10.1590/S0102311X2001000400028

Anjos, L. A., Rugan, R., Castro, E. M., \& Engstrom, A. M. (2003). Crescimento e estado nutricional em amostra probabilística de escolares no Município do Rio de Janeiro. Caderno Saúde Pública, 19(S1), S171-S179. doi: 10.1590/ S0102-311X2003000700018

Araújo, V. C., Graup, S., \& Pereira, E. F. (2007). Percepção da imagem corporal em relação ao estado nutricional em escolares. $6^{\circ}$ Fórum internacional de esportes. Universidade Federal de Santa Catarina. Consultado em 15 de setembro de 2009, a partir de http://www.unesporte.org. br/forum2007/apresentacao_oral/20_valberio_ araujo.pdf.

Bachelard, G. (1996). A formação do espírito científico: contribuição para uma psicanálise do conhecimento. Rio de Janeiro: Contraponto.

Balaban, G., Silva, G. A. P., \& Motta, M. E. F. A. (2001). Prevalência de sobrepeso e obesidade em escolares de diferentes classes socioeconômicas em Recife-PE. Pediatria, 23(4), 285-289. Consultado em 15 de setembro de 2009, a partir de http://pediatriasaopaulo.usp.br/upload/ pdf/537.pdf

Billington, C. J., \& Levine, A. S. (2000). Overweight, obesity, and health risk. Archives of Internal Medicine, 160(7), 898-904.

Bosi, M. L. M., \& Andrade, A. (2004). Transtornos do comportamento alimentar: um problema de saúde coletiva. Cadernos de Saúde Coletiva, 12(2), 197-202.

Braggion, G. F., Matsudo, S. M. M., \& Matsudo, V. K. R. (2000). Consumo alimentar, atividade física e percepção da aparência corporal em adolescentes. Revista Brasileira de Ciência e Movimento, $8(1), 15-21$.

Brougère, G. (2000). Brinquedo e cultura ( $3^{\mathrm{a}}$ ed.). São Paulo: Cortez.

Campos, L. A., Leite, A. J. M., \& Almeida, P. C. (2006). Nível socioeconômico e sua influência sobre a prevalência de sobrepeso e obesidade em escolares adolescentes do município de Fortaleza. Revista de Nutrição, 19(5), 531-538. doi: 10.1590/S1415-52732006000500001

Carvalho, A. M. P., Cataneo, C., Galindo, E. M. C., \& Malfará, C. T. (2005). Auto conceito e imagem corporal em crianças obesas. Paidéia, 15(30), 131-139. Consultado em 10 de outubro de 2009, a partir de http://sites.ffclrp.usp.br/ paideia/artigos/30/13.htm.

Carvalho, D., Paiva, A. A., Melo, A. S. O., Ramos, A. T., Medeiros, J. S., Medeiros, C. C. M., \& Cardoso, M. A. A. (2007). Perfil lipídico e estado nutricional de adolescentes. Revista Brasileira de Epidemiologia, 10(4), 491-498. doi: 10.1590/S1415-790X2007000400007

Cole, T. J., Bellizzi, M. C., Flegal, K. M., \& Dietz, W. H. (2000). Establishing a standard definition for child overweight and obesity worldwide: international survey. British Medical Journal, 320, 1240-1247. Consultado em 13 de outubro de 2009, a partir de http://www.ncbi.nlm.nih.gov/ pmc/articles/PMC27365/pdf/1240.pdf

Cole, T. J., Flegal, K. M., Nicholls, D., \& Jackson, A. A. (2007). Body mass index cut offs to define thinness in children and adolescents: international survey. British Medical Journal, 335, 194-202. doi:10.1136/bmj.39238.399444.55

Courtine, J. (1995). Os stakhanovistas do narcisismo: body-building e puritanismo ostentatório na cultura americana do corpo. In D. B. de Sant'Anna (Ed.), Políticas do corpo (pp. 81-114). São Paulo: Estação Liberdade.

Dowdell, E. B., \& Santucci, M. E. (2004). Health risk behavior assessment: nutrition, weight, and tobacco use in one urban seventh-grade class. Public Health Nursing, 21(2), 128-136. doi: 10.1111/j.0737-1209.2004.021206.x

Fernandes, R. A., Kawaguti, S. S., Agostini, L., Oliveira, A. R., Ronque, E. R. V., Freitas, J., \& Ismael, F. (2007). Prevalência de sobrepeso e obesidade em alunos de escolas privadas do município de Presidente Prudente - SP. Revista Brasileira de Cineantropometria e Desempenho Humano, 9(1), 21-27. Consultado em 17 de 
novembro de 2009, a partir de http://www. rbcdh.ufsc.br

Governo do Distrito Federal. (2009). Consultado em 17 de março de 2010, a partir de http://www. santamaria.df.gov.br/.

Hagger, M., Biddle, S., \& Wang, C. (2005). Physical self-concept in adolescence: generalizability of a multidimensional, hierarchical model across gender and grade. Educational and Psychological Measurement, 65(2), 297-322. doi: 10.1177/0013164404272484

Hills, A. P., King, N. A., \& Armstrong, T. P. (2007). The contribution of physical activity and sedentary behaviours to the growth and development of children and adolescents: implications for overweight and obesity. Sports Medicine, 37(6), 533-545.

Kann, L., Kinchen, S. A., Williams, B. I., Ross, J. G., Lowry, R., Grunbaum, J. A., \& Kolbe, L. J. (2000). Youth Risk Behavior Surveillance-United States, 1999. Journal of School Health, 70(7), 271-285. doi: 10.1111/j.17461561.2000.tb07252.x

Lohman, T. G., Roche, A. F., \& Martorell, R. (1988). Anthropometric standardization reference manual. Champaign, IL: Human Kinetics.

Maciel, F. T., \& Ferreira, J. S. (2010). Percepção da auto-imagem corporal em relação ao estado nutricional de escolares do ensino médio em Campo Grande, MS. Revista Digital de Buenos Aires, 146. Consultado em 10 de novembro de 2010, a partir de http://www.efdeportes.com/ efd146/auto-imagem-corporal-em-relacao-ao-estado-nutricional.htm.

Ministério da Saúde. (2009). VIGITEL - Vigilância de fatores de risco e proteção para doenças crônicas por inquérito telefônico. Consultado em 4 de abril de 2010 a partir de http:// portal.saude.gov.br/portal/arquivos/pdf/ vigitel2009_220610.pdf.

Ministério da Saúde. (2006). Secretaria de Vigilância em Saúde, Secretaria de Atenção à Saúde. Política nacional de promoção da saúde. Consultado em 4 de abril de 2010, a partir de http://portal. saude.gov.br/portal/arquivos/pdf/PNPS2.pdf.
Mondini, L., \& Monteiro C. A. (1998). Relevância epidemiológica da desnutrição e da obesidade em distintas classes sociais: métodos de estudo e aplicação à população brasileira. Revista Brasileira de Epidemiologia, 1(1), 14-20. doi: 10.1590/ S1415-790X1998000100004

Moreno, J., \& Cervelló, E. (2005). Physical self-perception in spanish adolescents: effects of gender and involvement in physical activity. Journal of Human Movement Studies, 48, 291-311. Consultado em 4 de novembro de 2009, a partir de http://www.um.es/univefd/self-concept.pdf

Murphy, J. M., Horton, N. J., Burke Jr, J. D., Monson, R. R., Laird, N. M., \& Lesage, A., Sobol, A. M. (2009). Obesity and weight gain in relation to depression: findings from the Stirling County Study Overeating while depressed. International Journal of Obesity, 33(3), 335-341. doi: 10.1038/ ijo.2008.273

Nunes, M. A., Olinto, M. T., Barros, F. C., \& Camey, S. (2001). Influência da percepção do peso e do índice de massa corporal nos comportamentos alimentares anormais. Revista Brasileira de Psiquiatria, 23(1), 21-27. Consultado em 13 de novembro de 2009, a partir de http://www. scielo.br/pdf/rbp/v23n1/a06v23n1.pdf

Ogden, C. L., Carroll, M. D., Curtin, L. R., McDowell, M. A., Tabak, C. J., \& Flegal, K. M. (2006). Prevalence of overweight and obesity in the United States, 1999-2004. Journal of the American Medical Association, 295(13), 1549-1555. doi: 10.1001/jama.295.13.1549

Oliveira, C. L., \& Fisberg, M. (2003). Obesidade na infância e adolescência - uma verdadeira epidemia. Arquivos Brasileiros de Endocrinologia E Metabologia, 47(2), 151-157. doi: 10.1590/ S0004-27302003000200001

Organização Mundial da Saúde (2000). World Health Organization consultation on obesity. Obesity: preventing and managing the global epidemic (WHO Technical Report Series 894). Consultado em 6 de dezembro de 2009, a partir de http://whqlibdoc.who.int/trs/who_trs_894.pdf. Palacios, J. (1995). O que é a adolescência. In C. Coll, J. Palacios \& A. Marchesi (Eds.), Desenvolvimento 
psicológico e educação: psicologia evolutiva (Vol. 1, pp. 263-272). Porto Alegre: Artes Médicas

Pinheiro, A. P., \& Giugliani, E. R. (2006). Who are the children with adequate weight who feel fat? Jornal de Pediatria, 82(3), 232-235. doi:10.2223/ JPED. 1477

Roemmich, J. N., Clark, P. A., Weltman A., \& Rogol A. D. (1997). Alterations in growth and body composition during puberty. I. Comparing multicompartment body composition models. Journal of Applied Physiology, 83(3), 927-935. Consultado em 4 de dezembro de 2009, a partir de http://jap.physiology.org/content/83/3/927. full.pdf + html

Sallis, J.F., Prochaska, J. J., \& Taylor, W. C. (2000). A review of correlates of physical activity of children and adolescents. Medicine \& Science in Sports \& Exercise, 32, 963-975. doi: 01959131/00/3205-0963/0

Schilder, P. (1999). A imagem do corpo: as energias construtivas da psique. São Paulo: Martins Fontes.

Silva, G. A. P., Balaban, G. N., Eulália, M. M., Baracho, J. D. S., \& Freitas, M. M. V. (2002). Prevalência de sobrepeso e obesidade em adolescentes de uma escola da rede pública do Recife. Revista Brasileira de Saúde Materno Infantil, 2(1), 37-42. doi: 0021-7557/01/77-02/96

Silva, K. S., Nahas, M. V., Hoefelmann, L. P., Lopes, A. S., \& Oliveira, E. S. (2008). Associações entre atividade física, índice de massa corporal e comportamentos sedentários em adolescentes. Revista Brasileira de Epidemiologia, 11 (1), 159-168. doi: 10.1590/S1415-790X2008000100015

Silva, M., \& Palmeira, A. L. (2010). Associações entre auto-conceito físico e motivação para o exercício em adolescentes: interações com o nível de prática e o gênero. Revista de Educação Física, 148(1), 227-236. Consultado em 4 de outubro de 2010, a partir de http://www.revistadeeducacaofisica.com.br/artigos/2010.1/005.pdf
Sisto, F. F. (2005). Aceitação-rejeição para estudar a agressividade na escola. Psicologia em Estudo, 10(1), 117-125. Consultado em 4 de novembro de 2009, a partir de http://www.scielo.br/pdf/ pe/v10n1/v10n1a13.pdf

Suñé, F. R., Dias-da-Costa, J. S., Olinto, M. T. A., \& Pattussi, M. P. (2007). Prevalência e fatores associados para sobrepeso e obesidade em escolares de uma cidade no Sul do Brasil. Caderno de Saúde Pública, 23(6), 1361-1371. Consultado em 3 de novembro de 2009, a partir de http://www. scielo.br/pdf/csp/v23n6/10.pdf

Tassitano, R. M., Bezerra, J., Tenório, C. M. C., Colares, V., Barros, M. V. G., \& Hallal, P. C. (2007). Atividade física em adolescentes brasileiros: uma revisão sistemática. Revista Brasileira de Cineantropometria e Desempenho Humano, 9(1), 55-60. Consultado em 3 de dezembro de 2009, a partir de http://journal.ufsc.br/index.php/ rbcdh/article/download/4033/3419

Wang, Y., Monteiro, C. A., \& Popkin, B. M. (2002). Trends of obesity and underweight in older children and adolescents in the United States, Brazil, China, and Russia. American Journal of Clinical Nutrition, 75(6), 971-977. Consultado em 3 de dezembro de 2009, a partir de http:// www.ajcn.org

Weiss, R., \& Kaufman, F. R. (2008). Metabolic complications of childhood obesity. Diabetes Care, 31, S310-S316. doi: 10.2337/dc08-s273

Wieting, M. J. (2008). Cause and effect in childhood obesity: solutions for a national epidemic. Journal of the American Osteopathic Association, 108(10), 545-552. Consultado em 10 de novembro de 2009, a partir de http://www.jaoa. org/content/108/10/545.full.pdf+html

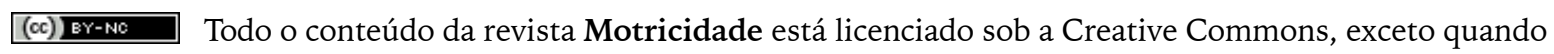
especificado em contrário e nos conteúdos retirados de outras fontes bibliográficas. 